\title{
Simulation of a balloon expandable stent in a realistic coronary artery-Determination of the optimum modelling strategy
}

\author{
Houman Zahedmanesh ${ }^{\text {a }}$, Daniel John Kelly ${ }^{\mathrm{b}}$, Caitríona Lally ${ }^{\mathrm{a}, \mathrm{b}, *}$ \\ a School of Mechanical and Manufacturing Engineering, Dublin City University, Dublin 9, Ireland \\ ${ }^{\mathrm{b}}$ Trinity Centre for Bioengineering, School of Engineering Trinity College Dublin, Ireland
}

\section{A R T I C L E I N F O}

\section{Article history:}

Accepted 31 March 2010

\section{Keywords:}

Balloon expandable stent

Finite element method

Arterial wall mechanics

Cardiovascular biomechanics

\begin{abstract}
A B S T R A C T
Computational models of stent deployment in arteries have been widely used to shed light on various aspects of stent design and optimisation. In this context, modelling of balloon expandable stents has proved challenging due to the complex mechanics of balloon-stent interaction and the difficulties involved in creating folded balloon geometries. In this study, a method to create a folded balloon model is presented and utilised to numerically model the accurate deployment of a stent in a realistic geometry of an atherosclerotic human coronary artery. Stent deployment is, however, commonly modelled by applying an increasing pressure to the stent, thereby neglecting the balloon. This method is compared to the realistic balloon expansion simulation to fully elucidate the limitations of this procedure. The results illustrate that inclusion of a realistic balloon model is essential for accurate modelling of stent deformation and stent stresses. An alternative balloon simulation procedure is presented however, which overcomes many of the limitations of the applied pressure approach by using elements which restrain the stent as the desired diameter is achieved. This study shows that direct application of pressure to the stent inner surface may be used as an optimal modelling strategy to estimate the stresses in the vessel wall using these restraining elements and hence offer a very efficient alternative approach to numerically modelling stent deployment within complex arterial geometries. The method is limited however, in that it can only predict final stresses in the stented vessel and not those occurring during stent expansion, in which case the balloon expansion model is required.
\end{abstract}

(c) 2010 Elsevier Ltd. All rights reserved.

\section{Introduction}

An estimated 652,000 percutaneous coronary interventions involving stents deployments were performed in the United States in 2006 to restore the blood supply to the hearts of patients suffering from cardiovascular diseases (American Heart Association (AHA), 2009). The majority of stents are delivered to the pathological region in a crimped configuration mounted on a folded balloon at the tip of a percutaneously inserted catheter and expanded in the stenosed region by inflation of the folded balloon. Stenting procedures have several significant advantages over open heart surgery, in particular the fact that it is a minimally invasive procedure, however, intimal cells can proliferate due to the severity of the injury caused to the arterial wall by the stent often leading to excessive neointimal hyperplasia and restenosis of the artery (Grewe et al., 2000; Mitra and Agrawal, 2006).

\footnotetext{
* Corresponding author at: School of Mechanical and Manufacturing Engineering, Dublin City University, Dublin 9, Ireland. Tel.: +3531 7007608; fax: +35317007148.

E-mail address: triona.lally@dcu.ie (C. Lally).
}

In recent years drug eluting stents, coated with anti-proliferative drugs, have reduced the restenosis rate compared to bare metal stents (Hara et al., 2006; Morice et al., 2002; Sousa et al., 2005; Serruys et al., 2006). Several mechanical factors linked to stent design and deployment have also been postulated to be involved in the development of this pathological condition however, which are not eliminated by such pharmaceutical interventions (McClean and Eigler, 2002; Timmins et al., 2007). The delivery of anti-proliferative drugs to surrounding tissue is also significantly influenced by the stent design (Hwang et al., 2001). Endothelial denudation and vessel wall injury during stent deployment and alteration of the stress and strain field in the arterial wall following stent deployment are hypothesised to be some of the most important factors contributing to in-stent restenosis (Kornowski et al., 1998; Mitra and Agrawal, 2006; Schwartz et al., 1992). Therefore, optimisation of stent design to improve mechanical performance is essential to achieve lower restenosis rates.

Computational models of stent deployment are excellent tools for optimisation of stent designs and can be used along with experimental studies to improve the mechanical performance of stents. In addition to being cost effective, computational models 
enable the stresses induced in the vessel wall to be estimated and therefore the degree of vascular injury and such models can therefore provide insights into the various aspects of stent design that may reduce the risk of arterial injury.

Many numerical studies have investigated the mechanical response of balloon expandable stents and suggested different strategies for their simulation (Auricchio et al., 2001; Gijsen et al., 2008; Holzapfel et al., 2005a; Lally et al., 2005; Lee et al., 1993; Migliavacca et al., 2005, 2007; Prendergast et al., 2003; Rogers et al., 1999; Wang et al., 2006; Zahedmanesh and Lally, 2009). Given the difficulties involved in construction of the model geometry and the complex contact problem involved in the interaction of a balloon, stent, and artery, many simplified methods have been used to model the complex mechanics of balloon expandable stent deployment. Balloons used for stent deployment are initially in a folded configuration and they consequently expand in a highly non-linear fashion when pressurised. This complex procedure of balloon unfolding is difficult and very computationally expensive to model.

Four main strategies have been used in the literature for numerically modelling balloon expansion of stents which include (i) direct application of a uniform pressure to the stent luminal surface (Dumoulin and Cochelin, 2000; De Beule et al., 2006; Early et al., 2008; Migliavacca et al., 2005; Zahedmanesh and Lally, 2009), (ii) rigid cylinder expansion using radial displacement (Hall and Kasper, 2006; Takashima et al., 2007; Wu et al., 2007), (iii) a folded balloon model (De Beule et al., 2008; Gervaso et al., 2008), and (iv) pressurisation of elastic cylinders with hyperelastic material properties (Ju et al., 2008; Kiousis et al., 2009). Gervaso et al. (2008) compared the first three strategies and concluded that a folded balloon model is necessary for accurate estimation of mechanical stresses. In spite of this, the computational costs and the effort involved in modelling folded balloons has urged many researchers to resort to alternative strategies which disregard the balloon model. In this context, Early et al. (2008) proposed a method of stent expansion by direct application of a uniform pressure on the inner stent surface in conjunction with special function elements connected to the stent nodes that restrained stent expansion as the desired diameter was achieved. However, no comparison with stent deployment using folded balloons was presented to fully support the use of this method and to elucidate its limitations.

In this study, stent deployment in a patient derived model of an atherosclerotic human coronary artery was simulated using the finite element method and a realistic model of a three-fold balloon. In addition, deployment of the same stent in the artery was simulated by direct application of uniform pressure to the inner stent surface, both with and without utilisation of special function restraining elements. The results from the three models, in particular the deformations and stresses in the vessels with fully deployed stents, were compared in an effort to investigate the optimal and most efficient method for numerically modelling stent deployment.

\section{Materials and methods}

A finite element model of a patient specific atherosclerotic coronary artery and the ACS Multi-Link RX DUET stent was constructed and the stent was expanded within the artery using three different strategies as defined by the following case studies:

(i) A uniformly increasing pressure, to a maximum value of 16 atm, was directly applied to the inner stent surface.

(ii) A uniformly increasing pressure was directly applied to the inner stent surface to achieve an inner stent diameter equal to the fully expanded balloon $(3.5 \mathrm{~mm})$ in conjunction with connector elements, which restrained the stent expansion beyond this diameter. One end of these connector elements was connected to the nodes on the stent outer surface and the other end was connected to the ground (i.e. the initial coordinates of the same node prior to deformation). (iii) Expansion of the stent using a realistic three-fold balloon inflated to a diameter of $3.5 \mathrm{~mm}$ using a maximum pressure of $16 \mathrm{~atm}$.

To generate the simulations pertaining to each case study, the finite element model required a number of inputs: namely the geometry of the stent, the atherosclerotic coronary artery, and the three-fold balloon; the material properties of the stent, artery, and balloon; the appropriate application of loading and boundary conditions, as described later.

\subsection{Model geometry}

A patient derived model of an atherosclerotic human coronary artery was constructed based on digitised 3D angiography images. The images were obtained using a method developed and described by Messenger et al. (2001) to obtain an accurate 3D geometry of the lumen of an atherosclerotic artery. The vessel wall dimensions were generated based on assigning a healthy arterial wall thickness of $0.5 \mathrm{~mm}$ at the proximal end of the vessel, upstream from the stenosed region, by offsetting the lumen contour by $0.5 \mathrm{~mm}$. This outer diameter was then extrapolated across the stenosed region of the vessel to define both healthy vessel wall and atherosclerotic plaque regions. The model was subsequently meshed assigning 6 elements through the thickness of the healthy artery wall and three elements through the plaque thickness. The vessel was divided into three layers: intima, media, and adventitia. The thickness of each arterial layer was discretised by two elements and each layer was assigned using ratios of adventitia, media, and intima to the wall thickness of $0.38,0.33$, and 0.29 , respectively, consistent with the data reported by Holzapfel et al. (2005b). Eight node linear brick, reduced integration elements with hourglass control (ABAQUS element type C3D8R) were used to mesh the atherosclerotic coronary artery. The total number of elements used to mesh the atherosclerotic coronary artery was 29,400 , chosen based on mesh sensitivity studies. All elements were checked to ensure that no distorted elements were generated and element distortion control was used to prevent inaccuracies arising from excessive distortion of elements in the contact regions.

A full three-dimensional finite element model of the stent was generated and meshed in ANSYS based on the ACS MultiLink RX DUET manufactured by Guidant/ Advanced Cardiovascular Systems as outlined in Zahedmanesh and Lally (2009).

In order to create the geometry of the three-fold crimped balloon, the geometry of a fully expanded balloon was generated in ProEngineer (Needham, MA, USA) with a diameter of $3.5 \mathrm{~mm}$ and was exported into ABAQUS (PROVIDENCE, RI, USA). The imported geometry was meshed using 4-node doubly curved shell elements with reduced integration and hourglass control (ABAQUS element type S4R). The balloon was then deflated in an explicit simulation by application of a negative pressure of $0.01 \mathrm{MPa}$ on its inner surface using general contact and with the proximal and distal ends fully constrained. Symmetry boundary conditions were applied on the balloon nodes at every $120^{\circ}$ on the balloon circumference, so as to deflate the balloon into a tri-wing configuration. The wings were folded circumferentially onto one another in a subsequent step by application of pressure to one side of each wing while the base of each wing was fully constrained generating a three-fold crimped balloon configuration, see Fig. 1.

\subsection{Material properties}

Layer specific human coronary arterial wall properties were assigned to the artery, consisting of the intima, media and adventitia and human atherosclerotic plaque as previously published in Zahedmanesh and Lally (2009).

The stress-strain response of the stent was applied based on data from Murphy et al. (2003) for 316 L stainless steel stent struts as previously defined in Zahedmanesh and Lally (2009).

The balloon was modelled as an isotropic, linear-elastic material, with a Young's modulus of $900 \mathrm{MPa}$ and Poisson's ratio of 0.3 based on the data published by Gervaso et al. (2008).

\subsection{Boundary conditions}

The load used for the expansion of the stents in case study (i) and (iii) was $16 \mathrm{~atm}$ which was applied on the inner surface of the stent and the inner balloon surface, respectively. This value is the maximum clinically permissible pressure for the expansion of most stents (Serruys and Kutryk, 1998, 2000). However in case study (ii), a maximum load of $30 \mathrm{~atm}$ was applied to the stent inner surface to fully expand the stent to a diameter equivalent to the diameter achieved using the balloon model. To ensure that the stent diameter did not exceed the balloon diameter due to the application of this clinically meaningless pressure, restraining connector elements were applied to every node of the stent. All of the restraining connector elements were attached to ground and assigned a length of $1.113 \mathrm{~mm}$ to ensure that the stent expanded from its crimped state to a final expansion diameter of $3.5 \mathrm{~mm}$.

In order to model the adhesion of the balloon to the catheter in case study (iii), the two end nodes of the balloon were connected using a connector element which was assigned a maximum length equal to the balloon length in the folded 

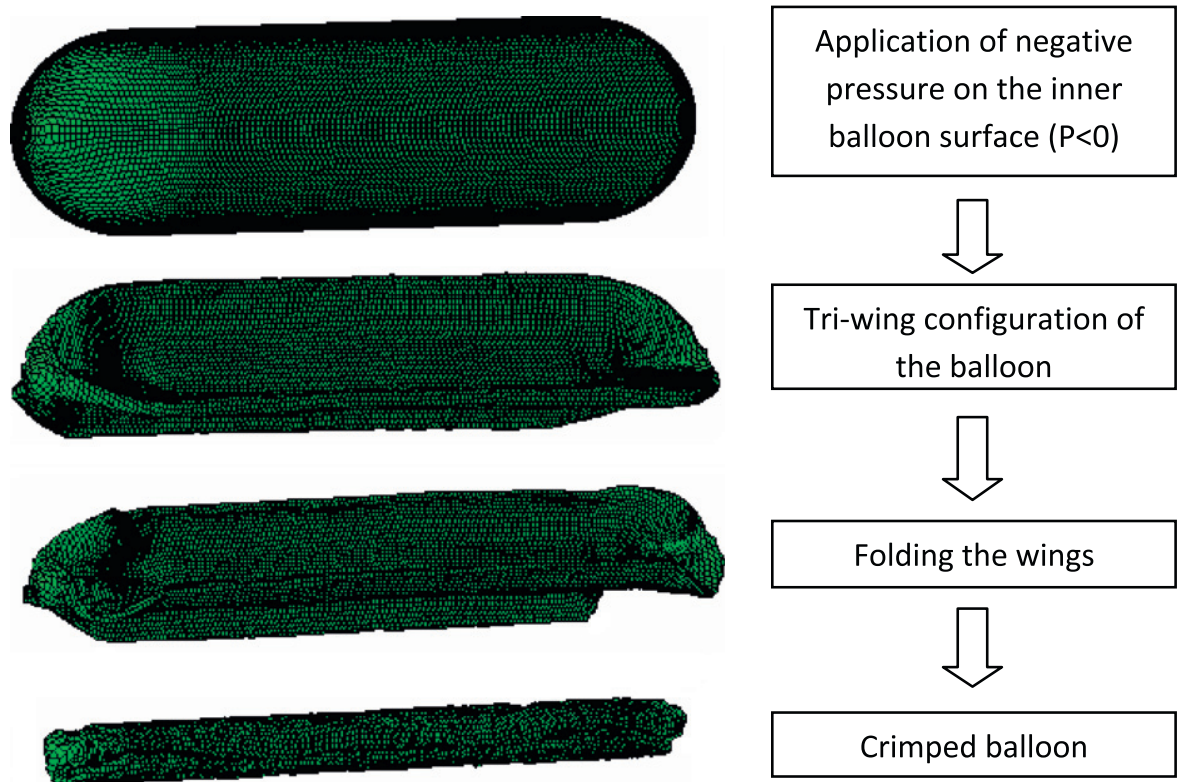

Fig. 1. The process of creating the three-fold balloon geometry.

configuration thus allowing for possible balloon bending. The end nodes of the balloon were constrained in the tangential direction. In addition, the two ends of the coronary artery were constrained in all models.

The general contact available in ABAQUS explicit was used to model the contact between the stent, balloon, and artery using frictionless contact with the default properties in all models. In addition, in all of the case studies three circumferentially symmetric nodes in the middle of the stents were constrained in the axial direction.

ABAQUS explicit was used in this study and when performing a quasi-static analysis using the explicit approach it is vital to ensure that the inertial forces are negligible throughout the simulation and do not cause unrealistic dynamic effects. Therefore, in this study the ratio of kinetic energy to the total strain energy was consistently maintained lower than $5 \%$. This criterion was proposed by Kim et al. (2002) and ensures that the dynamic effects are negligible. In order to respect this criterion the simulation times were set to $3 \mathrm{~s}$ in all case studies.

\section{Results}

Expansion of the three-fold balloon within the model of the coronary artery enabled a realistic simulation of stent deployment, see Fig. 2. Stent deformation was, however, considerably different in each of the three case studies. Deformation of the stent was investigated by studying the pressure-expansion response, flaring of the stent widely known as dog-boning, and foreshortening which represents the shortening of the stent length.

The overall pressure-diameter response in the mid-stent region in all case studies was determined as a function of pressure. Comparison of the curves illustrates a significantly different pressure-expansion response in case study (iii) in which the stent-balloon assembly showed a highly compliant response as the pressure reached $4 \mathrm{~atm}$ and the diameter increased rapidly from 1.5 to $3 \mathrm{~mm}$, see Fig. 3. This highly compliant response cannot be generated using direct application of a uniformly increasing pressure and this response is consistent with those reported in experimental studies of stent deployment (Kiousis et al., 2009; Migliavacca et al., 2008).

Dog-boning and foreshortening may be defined using the following (Kiousis et al., 2009):

Dogboning $(D B)=\frac{D_{\text {distal }}-D_{\text {central }}}{D_{\text {distal }}}$
Foreshortening $(F S)=\frac{L_{\text {deformed }}-L_{\text {undeformed }}}{L_{\text {deformed }}}$

where $D_{\text {distal }}$ and $D_{\text {central }}$ represent the diameter at distal and central locations of the stent, respectively; $L_{\text {deformed }}$ and $L_{\text {undeformed }}$ represent the deformed and undeformed length of the stent respectively.

In all case studies, dog-boning occurred upon pressurisation, however in case study (iii) where the stent was expanded using a balloon model, the dog-boning was more pronounced and initiated at a lower pressure, see Fig. 4. Maximum dog-boning in case study (iii) reached $49 \%$ at a pressure of 4 atm whilst in case studies (i) and (ii) this value reached a maximum of $19 \%$ at a pressure of $14.4 \mathrm{~atm}$. The final dog-boning after stent recoil however was identical in case studies (ii) and (iii).

The most significant difference in the stent deformation pattern, however, was found in the foreshortening curves, see Fig. 5. Case study (iii) shows a foreshortening response with negative values at all pressures, representing shortening of the stent, and reaching a maximum foreshortening of $-10 \%$ at a pressure of $5.6 \mathrm{~atm}$. In contrast, the other case studies show stent elongation. Experimental measurements on very similar stents have demonstrated stent shortening during stent expansion (Kiousis et al., 2009).

Exploring the stresses in the vessel wall showed that the highest stresses occurred in the plaque and intima. In case studies (ii) and (iii) the von Mises stresses induced in the arterial tissue were of very similar magnitudes with maximum values of approximately 950 and $170 \mathrm{kPa}$ in the plaque and intima, respectively, whilst the stresses in case study (i) were much lower, see Fig. 6. Moreover, the percentage of the tissue volume stressed over a certain threshold, calculated based on the stress value at the element integration points, proved very similar in case studies (ii) and (iii), see Fig. 7.

In addition, von Mises stress as high as $761 \mathrm{MPa}$ was found in the stent expanded using the balloon which was $10 \%$ and $4 \%$ higher than that predicted in case study (i) and (ii), respectively. These high stresses occurred where the crown joined the longitudinal struts and also in the crown tips in all cases. 
a

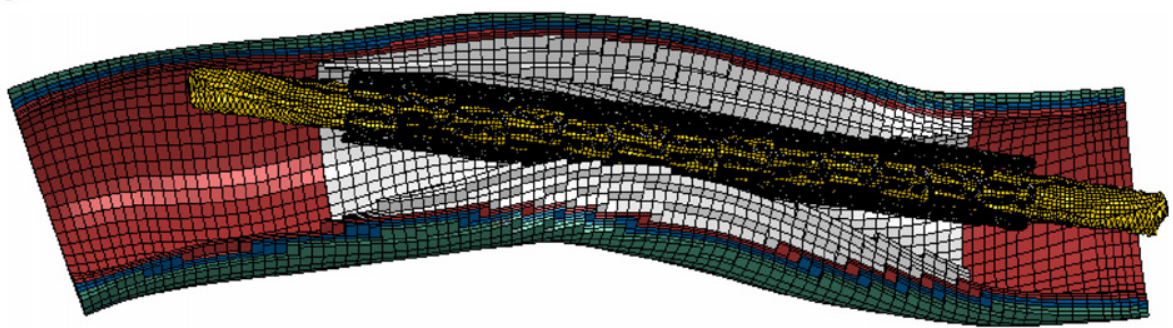

b

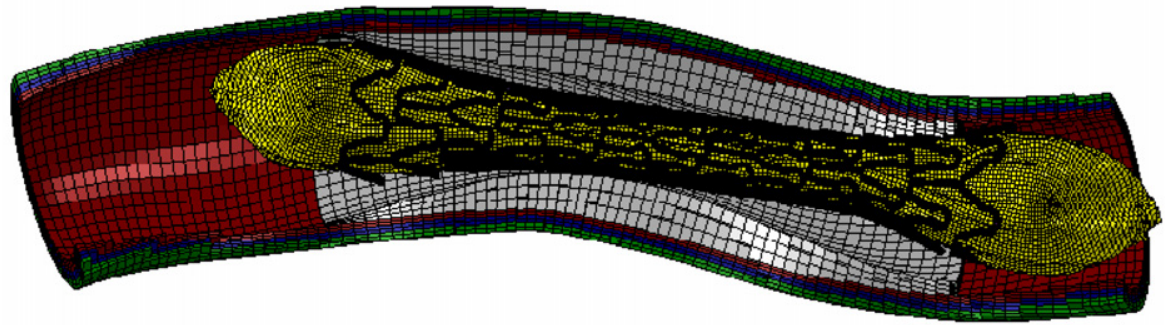

C

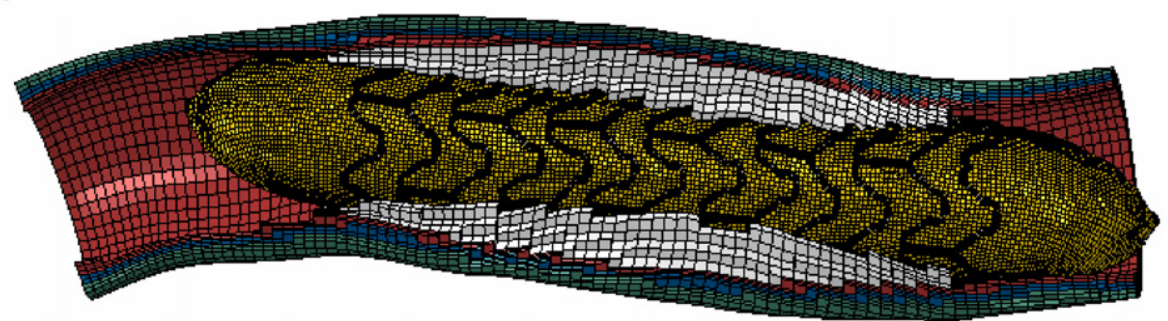

Fig. 2. Assembly of layer specific stenosed coronary artery, stent and balloon in (a) crimped configuration prior to expansion, (b) dog-boned configuration upon pressurisation, and (c) following full expansion. The adventitia, media, intima, and the atherosclerotic plaque are represented by green, blue, red, and grey, respectively. (For interpretation of the references to colour in this figure legend, the reader is referred to the web version of this article.)

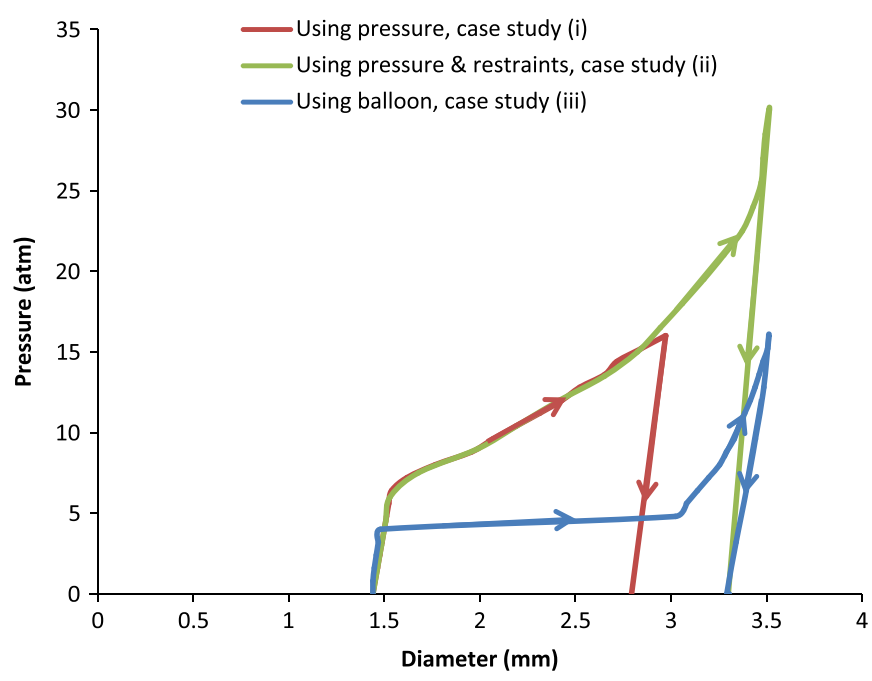

Fig. 3. Pressure-diameter relationship of the stent throughout the deployment process.

\section{Discussion}

Gervaso et al. (2008) compared simulation of stent deployment in an idealised vessel by direct application of pressure to the inner stent surface and also by using a folded balloon model and concluded that modelling the balloon is essential to accurately estimate the level of injury to the arterial wall. In another study by De Beule et al. (2008), the same two scenarios were compared in a free expansion simulation (without inclusion of any vessel)

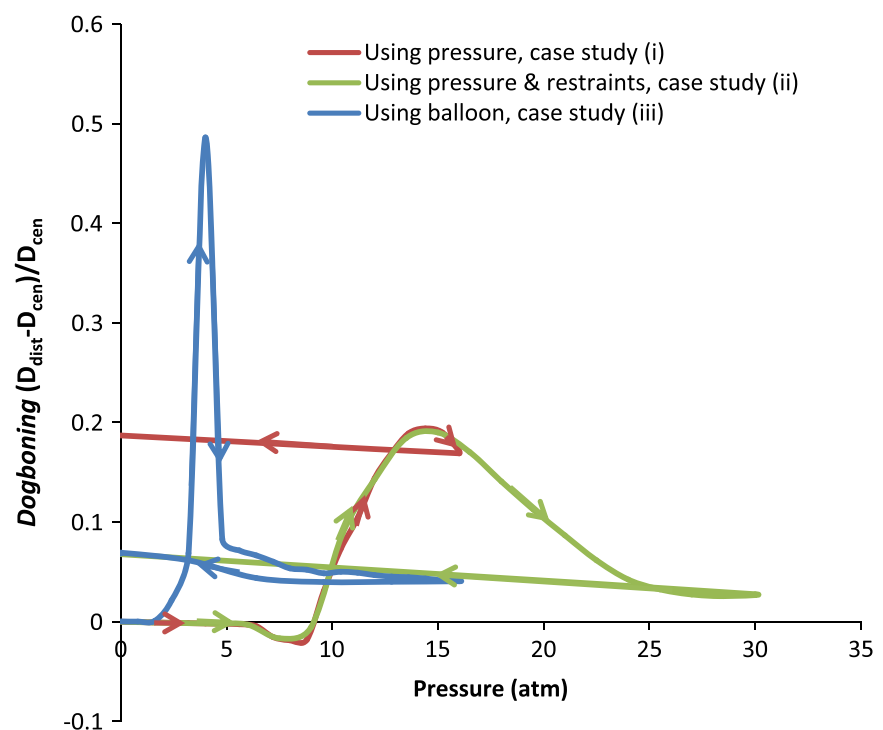

Fig. 4. Dog-boning of the stent as a function of the applied pressure throughout the deployment process.

and the pressurisation technique was found to be an over simplification which could not yield accurate results. Consistently, in this study comparison of case study (i) and case study (iii) illustrates that application of the clinically relevant pressures to the stent inner surface neither results in accurate estimation of the stress-strain field in the arterial wall nor is it able to predict 
the transient response, final deformed configuration and stresses of the stent.

The results of case study (ii), however, demonstrate that this scenario may have the potential to reasonably predict the stressstrain field in the vessel wall. This study illustrates that estimation of the transient response of the stent such as pressure-diameter, dog-boning, and foreshortening is clearly not possible by direct application of pressure to the stent inner surface and requires utilisation of a balloon model. Nevertheless in certain cases where the objective is the estimation of the final vessel wall stress/strain field following full stent expansion and recoil, use of the method outlined in case study (ii) may be adopted. In this study, a 6\% error in the estimation of the stent length on full expansion and recoil in case study (ii) did not cause any significant differences in the vessel wall stress magnitudes or spatial distribution. An additional limitation of this method that would benefit from further investigation is the degree to which it is influenced by the asymmetry of the vessel stenosis. Constraining the stent using restraining elements may result in a more

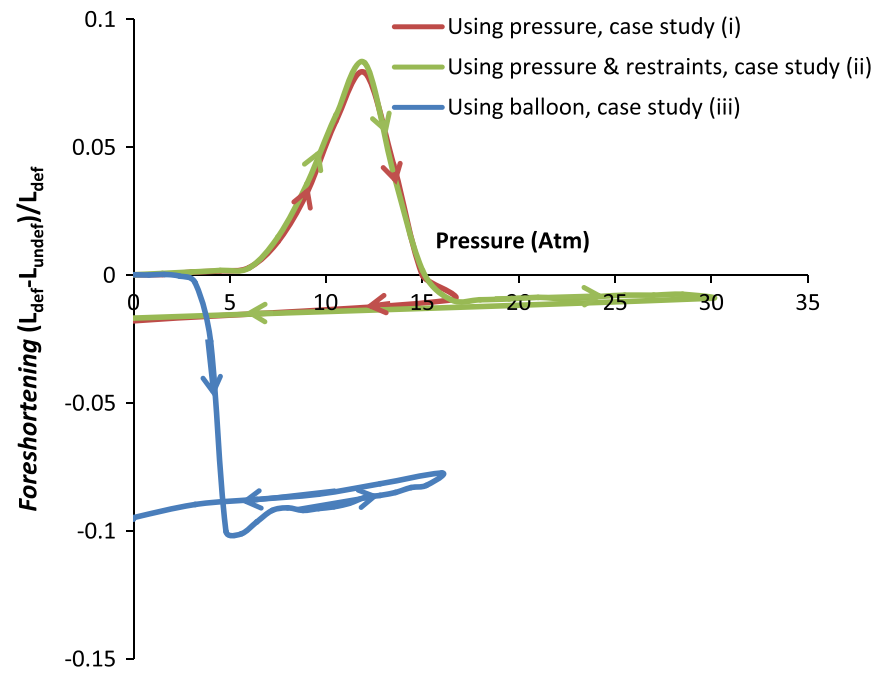

Fig. 5. Foreshortening of the stent as a function of applied pressure throughout the deployment process. symmetrical expansion than that which would occur with a balloon which is free to inflate in an asymmetrical configuration. The model geometry in this study has a lumen and stenosis of moderate asymmetry but expansion of stents using this method in vessels with more extreme asymmetry could lead to inaccuracies that would not occur using the balloon expansion model.

Although this study presents a simulation of a balloon expandable stent in a patient derived vessel geometry using a realistic three-fold balloon and a three layered vessel, some limitations remain in the models. The most important limitations of this study are associated with the vessel wall material properties. Damage to the vessel wall and residual stresses in the artery were not included in the constitutive model of the vessel. However, given that this is a comparative study which investigates the different modelling strategies, inclusion of damage and residual stresses in the vessel material model was deemed beyond the scope of this work. In addition, human coronary arteries generally exhibit an anisotropic response in the axial and circumferential directions due to collagen fibre orientation. The response of the vessel wall during stent expansion, however, is mainly dictated by its circumferential mechanical properties and therefore in this study the material properties of

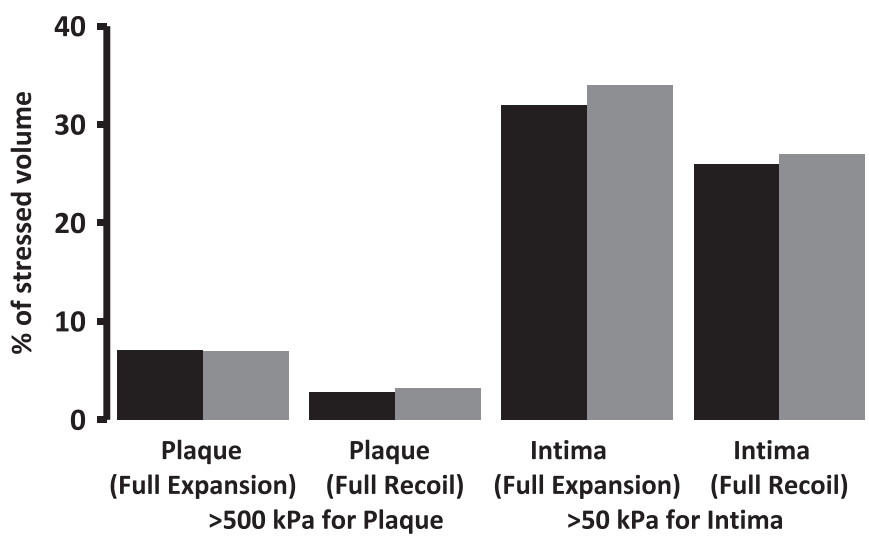

Fig. 7. Percentage volume of plaque tissue stressed above $500 \mathrm{kPa}$ and of intimal tissue stressed above $50 \mathrm{kPa}$ using uniform pressure with restraining elements (black) and a folded balloon (grey).
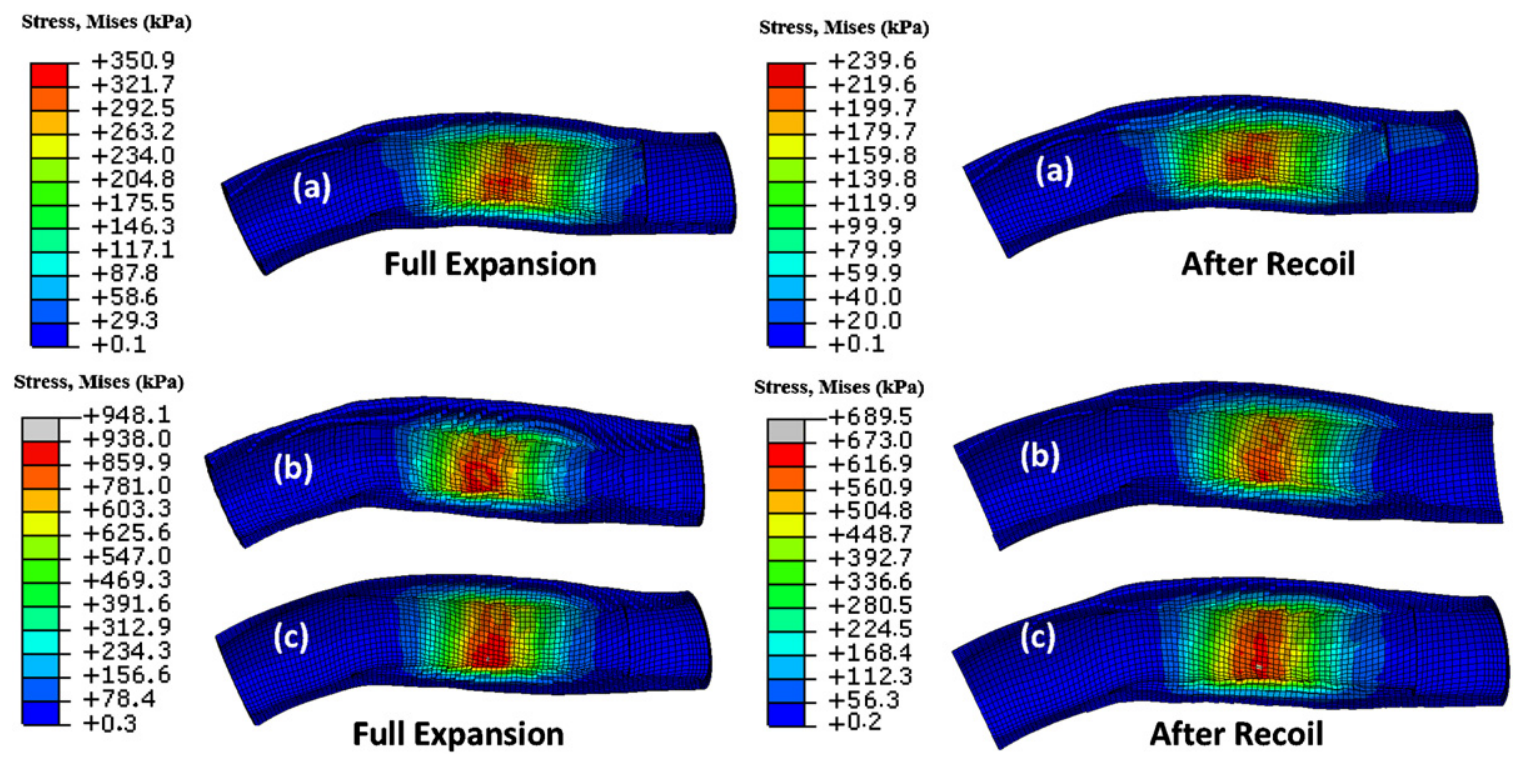

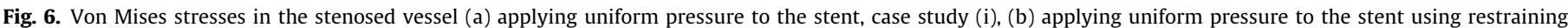
elements, case study (ii), and (c) using the balloon model, case study (iii). 
human coronary arterial layers in the circumferential direction were assigned to the models using an isotropic material model.

Although no experimental studies were carried out in this work to verify the numerical results obtained, the experimental work carried out by Kiousis et al. (2009) on similar stent designs shows pressure-diameter, dog-boning and foreshortening responses consistent with the results of case study (iii) and provide clear evidence to support the accuracy of this approach.

\section{Conclusions}

This study presents a realistic simulation of the deployment of a balloon expandable stent in a human atherosclerotic coronary artery using a realistic three-fold balloon model and compares the results to those obtained using the simplified modelling approach of direct application of pressure to the inner stent surface. Application of direct pressure to the inner stent surface was found to be incapable of accurately predicting the stress-strain field and the deformed configuration of both the stent and artery. In contrast however, application of pressure with restraining elements which prevent expansion of the stent beyond the desired diameter, may be used as a computationally efficient method to accurately predict the stress-strain field in the vessel wall following full stent expansion and recoil.

\section{Conflict of interest}

There is no conflict of interest to be declared by the authors.

\section{Acknowledgments}

The authors would like to acknowledge the contribution of Prof. Messenger's group at Denver V A Medical Center, Denver, Colorado, USA, for providing the coronary artery lumen surface contours obtained from 3D angiographic data which formed the basis for the realistic atherosclerotic geometry developed for this simulation.

This work was funded by the Irish Research Council for Science Engineering and Technology (IRCSET) under an Embark Initiative postgraduate scholarship.

\section{References}

American Heart Association, 2009. Heart Disease and Stroke Statistics 2009 American Heart Association, Dallas, TX.

Auricchio, F., Di Loreto, M., Sacco, E., 2001. Finite-element analysis of a stenotic revascularization through a stent insertion. Computer Methods in Biomechanics and Biomedical Engineering 4, 249-264

Dumoulin, C., Cochelin, B., 2000. Mechanical behaviour modelling of balloonexpandable stents. Journal of Biomechanics 33, 1461-1470.

De Beule, M., Van Impe, R., Verhegghe, B., Segers, P., Verdonck, P., 2006. Finite element analysis and stent design: reduction of dogboning. Technology and Health Care 14, 233-241.

De Beule, M., Mortier, P., Carlier, S.G., Verhegghe, B., Van Impe, R., Verdonck, P., 2008. Realistic finite element-based stent design: the impact of balloon folding. Journal of Biomechanics 41, 383-389.

Early, M., Lally, C., Prendergast, P.J., Kelly, D.J., 2008. Stresses in peripheral arteries following stent placement: a finite element analysis. Computer Methods in Biomechanics and Biomedical Engineering 12, 25-33.

Gervaso, F., Capelli, C., Petrini, L., Lattanzio, S., Virgilio, L.D., Migliavacca, F, 2008. On the effects of different strategies in modelling balloon-expandable stenting by means of finite element method. Journal of Biomechanics 41, 1206-1212.

Gijsen, F.J.H., Migliavacca, F., Schievano, S., Socci, L., Petrini, L., Thury, A., Wentzel, J.J., van der Steen, A.F.W., Serruys, P.W.S., Dubini, G., 2008. Simulation of stent deployment in a realistic human coronary artery. BioMedical Engineering OnLine 6, 7-23.

Grewe, P.H., Deneke, T., Machraoui, A., Barmeyer, J., Muller, K.M., 2000. Acute and chronic tissue response to coronary stent implantation: pathologic findings in human specimens. Journal of American College of Cardiology 35, 157-163.

Hall, G.J., Kasper, E.P., 2006. Comparison of element technologies for modelling stent expansion. Journal of Biomechanical Engineering 128, 751-756.

Hara, H., Nakamura, M., Palmaz, J.C., Schwartz, R.S., 2006. Role of stent design and coatings on restenosis and thrombosis. Advanced Drug Delivery Reviews 58, 377-386.

Holzapfel, G.A., Stadler, M., Gasser, T.C., 2005a. Changes in the mechanical environment of stenotic arteries during interaction with stents: computational assessment of parametric stent designs. Journal of Biomechanical Engineering $127,166-180$

Holzapfel, G.A., Sommer, G., Gasser, C.T., Regitnig, P., 2005b. Determination of layer-specific mechanical properties of human coronary arteries with nonatherosclerotic intimal thickening and related constitutive modeling. American Journal of Physiology-Heart and Circulatory Physiology 289, H2048-H2058.

Hwang, C.W., Wu, D., Edelman, E.R., 2001. Physiological transport forces govern drug distribution for stent-based delivery. Circulation 104, 600-605.

Ju, F., Xia, Z., Sasaki, K., 2008. On the finite element modelling of balloonexpandable stents. Journal of the Mechanical Behavior of Biomedical Materials 1, 86-95.

Kim, J., Kang, Y.H., Choi, H.H., Hwang, S.M., Kang, B.S., 2002. Comparison of implicit and explicit finite-element methods for the hydroforming process of an automobile lower arm. International Journal of Advanced Manufacturing Technology 20, 407-413.

Kiousis, D.E., Wulff, A.R., Holzapfel, G.A., 2009. Experimental studies and numerical analysis of the inflation and interaction of vascular balloon catheter-stent systems. Annals of Biomedical Engineering 37, 315-330.

Kornowski, R., Hong, M.K., Tio, F.O., Bramwell, O., Wu, H., Leon, M.B., 1998. In-stent restenosis: contributions of inflammatory responses and arterial injury to neointimal hyperplasia. Journal of the American College of Cardiology 31, 224-230.

Lally, C., Dolan, F., Prendergast, P.J., 2005. Cardiovascular stent design and vessel stresses: a finite element analysis. Journal of Biomechanics 38, 1574-1581.

Lee, R.T., Loree, H.M., Cheng, G.C., Lieberman, E.H., Jaramillo, N., Schoen, F.J., 1993. Computational structural analysis based on intravascular ultrasound imaging before in vitro angioplasty: prediction of plaque fracture locations. Journal of the American College of Cardiology 21, 777-782.

McClean, R., Eigler, N.L., 2002. Stent design: implications for restenosis. Reviews in Cardiovascular Medicine 3, S16-S22.

Messenger, J.C., Chen, S.Y., Carroll, J.D., Burchenal, J.E., Kioussopoulos, K., Groves, B.M., 2001. 3D coronary reconstruction from routine single-plane coronary angiograms: clinical validation and quantitative analysis of the right coronary artery in 100 patients. International Journal of Cardiac Imaging 16, 413-427.

Migliavacca, F., Petrini, L., Montanari, V., Quagliana, I., Auricchio, F., Dubini, G., 2005. A predictive study of the mechanical behaviour of coronary stents by computer modelling. Medical Engineering and Physics 27, 13-18.

Migliavacca, F., Gervaso, F., Prosi, M., Zunino, P., Minisini, S., Formaggia, L., Dubini, G., 2007. Expansion and drug elution model of a coronary stent. Computer Methods in Biomechanics and Biomedical Engineering 10, 63-73.

Mitra, A.K., Agrawal, D.K., 2006. In stent restenosis: bane of the stent era. Journal of Clinical Pathology 59, 232-239.

Morice, J.C., Serruys, P.W., Sousa, J.E., et al., 2002. A randomized comparison of a sirolimus eluting stent with a standard stent for coronary revascularization. New England Journal of Medicine 346, 1773-1780.

Murphy, B.P., Savage, P., McHugh, P.E., Quinn, D.F., 2003. The stress-strain behaviour of coronary stent struts is size dependent. Annals of Biomedical Engineering 31, 686-691.

Prendergast, P.J., Lally, C., Daly, S., Reid, A.J., Lee, T.C., Quinn, D., Dolan, F., 2003. Analysis of prolapse in cardiovascular stents: a constitutive equation for vascular tissue and finite-element modelling. Journal of Biomechanical Engineering 125, 692-699.

Rogers, C., Tseng, D.Y., Squire, J.C., Edelman, E.R., 1999. Balloon-artery interactions during stent placement: a finite element analysis approach to pressure, compliance, and stent design as contributors to vascular injury. Circulation Research 84, 378-383.

Serruys, P.W., Kutryk, M.J.B., 1998. Handbook of Coronary Stents 2nd ed. Martin Dunitz Ltd, London.

Serruys, P.W., Kutryk, M.J.B., 2000. Handbook of Coronary Stents 3rd ed. Martin Dunitz Ltd, London.

Serruys, P.W., Kutryk, M.J.B., Ong, A.T.L., 2006. Coronary-artery stents-drug therapy. New England Journal of Medicine 354, 483-495.

Schwartz, R.S., Huber, K.C., Murphy, J.G., Edwards, W.D., Camrud, A.R., Vlietstra, R.E. Holmes, D.R., 1992. Restenosis and the proportional neointimal response to coronary artery injury: results in a porcine model. The American Journal of Cardiology 19, 267-274.

Sousa, J.E., Costa, M.A., Abizaid, A., et al., 2005. Four-year angiographic and intravascular ultrasound follow-up of patients treated with sirolimus-eluting stents. Circulation 111, 2326-2329.

Takashima, K., Kitou, T., Mori, K., Ikeuchi, K., 2007. Simulation and experimental observation of contact conditions between stents and artery models. Medical Engineering and Physics 29, 326-335.

Timmins, L.H., Moreno, M.R., Meyer, C.A., Criscione, J.C., Rachev, A., Moore Jr., J.E., 2007. Stented artery biomechanics and device design optimization. Medical and Biological Engineering and Computing 45, 505-513. 
Wang, W.Q., Liang, D.K., Yang, D.Z., Qi, M., 2006. Analysis of the transient expansion behavior and design optimization of coronary stents by finite element method. Journal of Biomechanics 39, 21-32.

Wu, W., Wang, W.Q., Yang, D.Z., Qi, M., 2007. Stent expansion in curved vessel and their interactions: a finite element analysis. Journal of Biomechanics 40, 2580-2585.
Zahedmanesh, H., Lally, C., 2009. Determination of the influence of stent strut thickness using the finite element method: implications for vascular injury and in-stent restenosis. Medical and Biological Engineering and Computing 47, 385-393. 